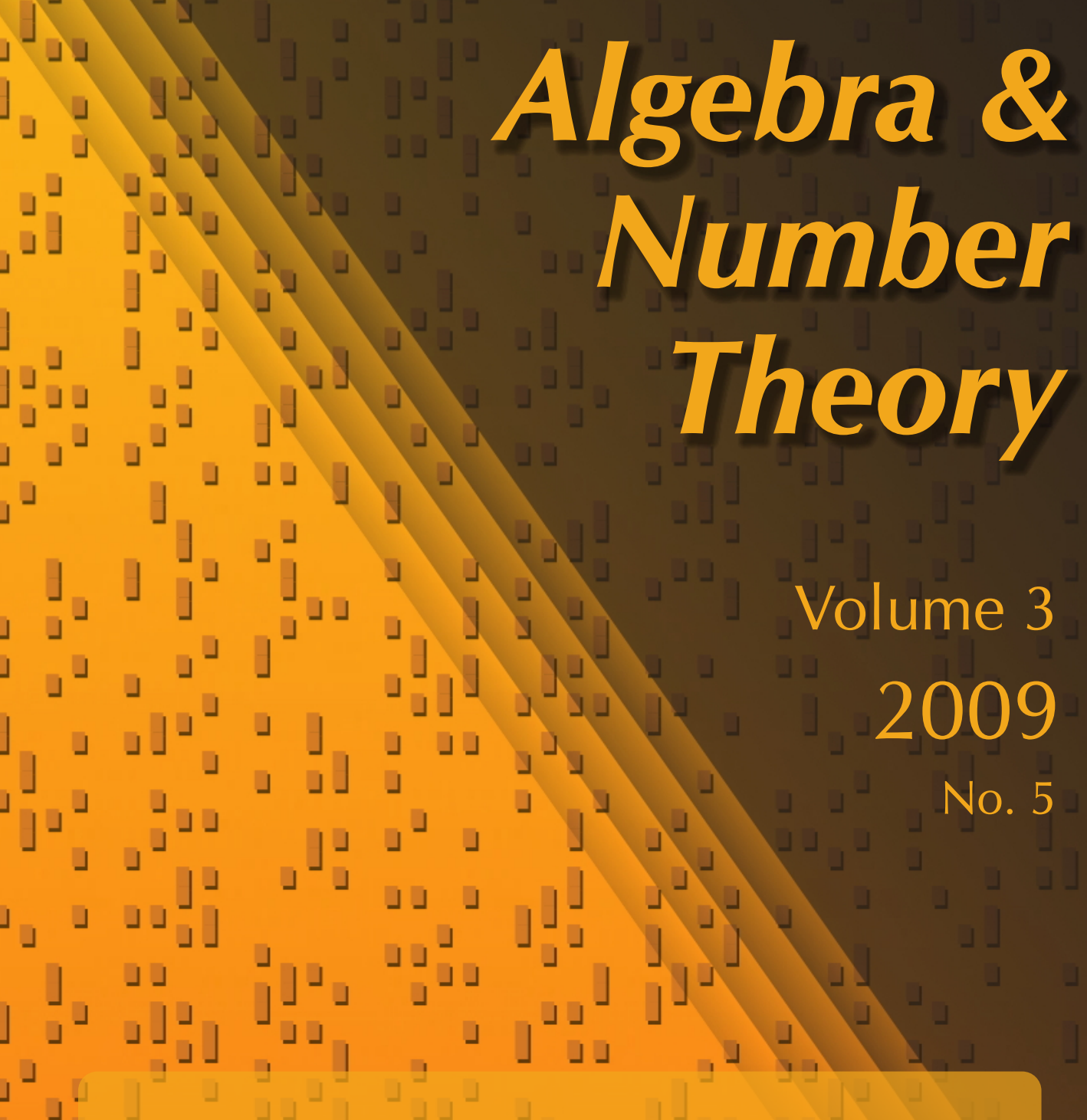

\title{
Compatible associative products and trees
}

Vladimir Dotsenko

$$
\begin{aligned}
& \text { دل } \\
& \lrcorner\lrcorner\lrcorner \quad\lrcorner\lrcorner\lrcorner\lrcorner\lrcorner\lrcorner\lrcorner\lrcorner\lrcorner\lrcorner\lrcorner\lrcorner
\end{aligned}
$$




\title{
Compatible associative products and trees
}

\author{
Vladimir Dotsenko
}

\begin{abstract}
We compute dimensions and characters of the components of the operad of two compatible associative products and give an explicit combinatorial construction of the corresponding free algebras in terms of planar rooted trees.
\end{abstract}

\section{Introduction}

Description of results. An algebra with two compatible associative products is a vector space $V$ equipped with two binary operations such that each of them is an associative product and these two products are compatible (that is, any linear combination of these products is again an associative product). Such algebras were recently studied by Odesskii and Sokolov [2006], who classified simple finitedimensional algebras of this type. In this paper, we study another extreme case: free algebras of this type. Namely, we compute dimensions of graded components of this algebra, and also give an interpretation of operations in terms of combinatorics of planar rooted trees.

Just as for an arbitrary algebraic structure, to get information about free algebras, one first computes the $S_{n}$-module structure (with respect to the action by permutations of the generators) on the "multilinear part" (that is, the space of elements in which each of the generators occurs exactly once) of the free algebra with $n$ generators. Then this information is used in a rather straightforward way to compute the dimensions of all graded components.

As our computation shows the free 1-generated algebra with two compatible products has Catalan numbers as dimensions of its graded components. We provide a materialisation of this formula describing two compatible products on planar rooted trees and proving that the algebra of planar rooted trees is a free algebra with two compatible products. (We actually give a more general construction which is valid for any number of generators.) We use this construction to obtain yet another proof of the results on the Grossman-Larson algebra of planar rooted trees.

MSC2000: primary 08B20; secondary 18D50, $05 \mathrm{C} 05$.

Keywords: operads, Koszul duality, compatible structures.

The author was partially supported by CNRS-RFBR grant no. 07-01-92214, by the President of the Russian Federation grant no. NSH-3472.2008.2 and by an IRCSET research fellowship. 
Machinery. To compute dimensions and characters for spaces of multilinear elements, we use Koszul duality for operads and the theory of Koszul operads developed by V. Ginzburg and M. Kapranov. It turns out that the Koszul dual to the operad of two compatible products is much simpler than the original operad. For any Koszul operad, information on the dimensions of its components can be used to obtain similar information for the dual operad. Namely, the following assertion is true.

Proposition 1.1 [Ginzburg and Kapranov 1994]. Let $f_{\mathscr{O}}(x):=\sum_{n=1}^{\infty} \frac{\operatorname{dim} \mathscr{O}(n)}{n !} x^{n}$. If $\mathscr{O}$ is a Koszul operad, then

$$
f_{\mathscr{O}}\left(-f_{\mathscr{O} !}(-x)\right)=x
$$

A similar functional equation holds for the generating functions of characters of representations of the symmetric groups in the components of an operad.

Example 1.2. For the associative operad As, we have $\operatorname{dim} \operatorname{As}(n)=n$ !, and thus $f_{\text {As }}(x)=x /(1-x)$. This operad is Koszul and self-dual, which agrees with the functional equation

$$
\frac{\frac{x}{1+x}}{1-\frac{x}{1+x}}=x .
$$

Koszulness of the operad of two compatible products was proved in [Strohmayer 2008], and this result is crucial for us. In our study of free algebras over $\mathrm{As}^{2}$, we use a simple but very elegant idea [Chapoton 2007; Fresse 1998] which applies in many cases when one wants to prove that some class of algebras consists of free algebras.

Outline of the paper. Throughout the paper, we assume that the reader is familiar with the main notions of operad theory. Still we briefly remind the reader of some of them when they appear in the text.

In Section 2, we recall some standard definitions of operad theory, define the operad of two compatible brackets, and list necessary facts about Koszul duality for operads. In Section 3, we compute the generating functions for the characters of our operads using functional equations on these generating functions and use them to identify the corresponding representations. In Section 4, we construct a monomial basis in the multilinear part of the free algebra with two compatible products. In Section 5, we prove that free algebras with two compatible products are free as associative algebras. In Section 6, we relate compatible associative products to combinatorics of trees. It turns out that the linear span of planar rooted trees has two compatible associative products and that the resulting algebra is a free algebra with two compatible products. We also give another proof of the result of [Grossman 
and Larson 1989] on the algebra of planar rooted trees. We conclude with some remarks and conjectural generalisations for other compatible structures.

All vector spaces and algebras throughout this paper are defined over an arbitrary field of zero characteristic.

\section{Operads: summary}

S-modules and operads. An S-module is a collection $\{\mathscr{V}(n), n \geq 1\}$ of vector spaces, where each $\mathscr{V}(k)$ is an $S_{k}$-module. Morphisms, direct sums, tensor products and duals of such objects are defined in the most straightforward way.

The module Det, where $\operatorname{Det}(n)$ is the sign representation of $S_{n}$, is an important example of an $\mathbb{S}$-module. We need the following version of the dual module: $\mathscr{V}^{\vee}=\mathscr{V}^{*} \otimes$ Det; this is the ordinary dual twisted by the sign representation. In some cases, we consider differential graded $S$-modules; all preceding constructions are defined for them in a similar way. The graded analogue of Det is denoted by $\mathscr{E}$; the space $\mathscr{E}(n)_{1-n}$ is one-dimensional and is the sign representation of the symmetric group, while all other spaces $\mathscr{E}(n)_{k}$ are zero.

Each $\mathbb{S}$-module $\mathscr{V}$ gives rise to a functor from the category $\mathscr{F} i n$ of finite sets (with bijections as morphisms) to the category of vector spaces. Namely, for a set $I$ of cardinality $n$ let

$$
\mathscr{V}_{I}=\mathbb{k} \operatorname{Hom}_{\mathscr{F} i n}([n], I) \otimes_{\mathbb{k} S_{n}} \mathscr{V}(n) .
$$

(Here $[n]$ stands for the "standard" set $\{1,2, \ldots, n\}$.) This space is often denoted by $\mathscr{V}(I)$; we prefer to use a different notation to avoid confusion with free algebras later.

For $\mathbb{S}$-modules $\mathscr{V}$ and $\mathscr{W}$, define the composition $\mathscr{V} \circ \mathscr{W}$ as

$$
(\mathscr{V} \circ \mathscr{W})(n)=\bigoplus_{m=1}^{n} \mathscr{V}(m) \otimes_{\mathbb{k} S_{m}}\left(\bigoplus_{f:[n] \rightarrow[m]} \bigotimes_{l=1}^{m} \mathscr{W}_{f^{-1}(l)}\right),
$$

where the sum is taken over all surjections $f$. This operation equips the category of $\mathbb{S}$-modules with a structure of a monoidal category. An operad is a monoid in this category. See [Markl et al. 2002] for a more detailed definition. To simplify the definitions, we consider in this paper only operads $\mathscr{O}$ with $\mathscr{O}(1)=\mathbb{k}$.

Let $V$ be a vector space. By definition, the operad $\operatorname{End}_{V}$ of linear mappings is the collection $\left\{\operatorname{End}_{V}(n)=\operatorname{Hom}\left(V^{\otimes n}, V\right), n \geq 1\right\}$ of all multilinear mappings of $V$ into itself with the obvious composition maps.

Using the operad of linear mappings, we can define an algebra over an operad $\mathscr{O}$; a structure of such an algebra on a vector space is a morphism of the operad $\mathscr{O}$ into the corresponding operad of linear mappings. Thus an algebra over an operad $\mathscr{O}$ is a vector space $W$ together with a collection $\mathscr{O}(n) \otimes_{\mathbb{k} S_{n}} W^{\otimes n} \rightarrow W$ of mappings 
with obvious compatibility conditions. The free algebra generated by a vector space $X$ over an operad $\mathscr{O}$ is

$$
\mathscr{O}(X):=\mathscr{O} \circ X=\bigoplus_{k=1}^{\infty} \mathscr{O}(n) \otimes_{\mathbb{k} S_{n}} X^{\otimes n} .
$$

Operads defined by generators and relations. The free operad $\mathscr{F}_{\mathscr{G}}$ generated by an $\mathbb{S}$-module $\mathscr{G}$ (with $\mathscr{G}(1)=0$ ) is defined as follows. A basis in this operad consists of some species of trees. These trees have a distinguished root (of degree one). A tree belonging to $\mathscr{F}_{\mathscr{G}}(n)$ has exactly $n$ leaves, its internal vertices (neither leaves nor the root) labelled by basis elements of $\mathscr{G}$, any vertex with $k$ siblings being labelled by an element of $\mathscr{G}(k)$. The unique tree whose set of internal vertices is empty generates the one-dimensional space $\mathscr{F}_{\mathscr{G}}(1)$. The composition of a tree $t$ with $l$ leaves and trees $t_{1}, \ldots, t_{l}$ glues the roots of $t_{1}, \ldots, t_{l}$ to the respective leaves of $t$. (In every case, two edges glued together become one edge, and the common vertex becomes an interior point of this edge.)

Free operads are used to define operads by generators and relations. Let $\mathscr{G}$ be an $\mathbb{S}$-module, and let $\mathscr{R}$ be an $\mathbb{S}$-submodule in $\mathscr{F} \mathscr{G}$. An (operadic) ideal generated by $\mathscr{R}$ in $\mathscr{F}_{\mathscr{G}}$ is the linear span of all trees such that at least one internal vertex is labelled by an element of $\mathscr{R}$. An operad with generators $\mathscr{G}$ and relations $\mathscr{R}$ is the quotient of the free operad $\mathscr{F}_{\mathscr{G}}$ modulo this ideal.

Definition 2.1. The associative operad As is generated by one binary operation $\star: a, b \mapsto a \star b$. The relations in this operad are equivalent to the associativity condition for every algebra over this operad:

$$
(a \star b) \star c=a \star(b \star c) .
$$

The operad of two compatible associative products $\mathrm{As}^{2}$ is generated by two binary operations (products) $\star_{1}$ and $\star_{2}$. The relations in this operad are equivalent to the following identities in each algebra over this operad: the associativity conditions

$$
\left(a \star_{1} b\right) \star_{1} c=a \star_{1}\left(b \star_{1} c\right) \text { and }\left(a \star_{2} b\right) \star_{2} c=a \star_{2}\left(b \star_{2} c\right)
$$

for products and the four-term relation

$$
\left(a \star_{1} b\right) \star_{2} c+\left(a \star_{2} b\right) \star_{1} c=a \star_{1}\left(b \star_{2} c\right)+a \star_{2}\left(b \star_{1} c\right)
$$

between the products.

Koszul duality for operads. Let an operad $\mathscr{O}$ be defined by a set of binary operations $\mathscr{B}$ with quadratic relations $\mathscr{R}$ (that is, relations involving ternary operations obtained by compositions from the given binary operations). In this case, $\mathscr{O}$ is said to be quadratic. For quadratic operads, there is an analogue of Koszul duality for quadratic algebras. To a quadratic operad $\mathscr{O}$, this duality assigns the operad $\mathscr{O} !$ with generators 
$\mathscr{B}^{\vee}$ and with the annihilator of $\mathscr{R}$ under the natural pairing as the space of relations. Just as in the case of quadratic algebras, $(\mathscr{O} !) ! \simeq \mathscr{O}$.

Example 2.2 [Ginzburg and Kapranov 1994]. The operad As is self-dual:

$$
\mathrm{As}^{!} \simeq \mathrm{As} .
$$

The cobar complex $\mathbf{C}(\mathscr{O})$ of an operad $\mathscr{O}$ is the free operad with generators $\left\{\mathscr{O}^{*}(n), n \geq 2\right\}$ equipped with a differential $d$ with $d^{2}=0$ (see [Markl et al. 2002] for details). Once again we use twisting by the sign, now to get another version of the cobar complex, $\mathbf{D}(\mathscr{O})=\mathbf{C}(\mathscr{O}) \otimes \mathscr{E}$. The zeroth cohomology of $\mathbf{D}(\mathscr{O})$ is isomorphic to the operad $\mathscr{O}$ !.

Definition 2.3. An operad $\mathscr{O}$ is said to be Koszul if $H^{i}(\mathbf{D}(\mathscr{O}))=0$ for $i \neq 0$.

Proposition 2.4 [Strohmayer 2008]. The operad As ${ }^{2}$ is Koszul.

Generating functions and characters. As we mentioned above, to each operad (and more generally, to each $\mathbb{S}$-module) $\mathscr{O}$ one can assign the formal power series (the exponential generating function of the dimensions)

$$
f_{\mathscr{O}}(x)=\sum_{n=1}^{\infty} \frac{\operatorname{dim} \mathscr{O}(n)}{n !} x^{n},
$$

and if $\mathscr{O}$ is a Koszul operad, then $f_{\mathscr{O}}\left(-f_{\mathscr{O}}(-x)\right)=x$.

This functional equation is an immediate corollary of a functional equation relating more general generating functions that will be defined now.

The character of a representation $M$ of the symmetric group $S_{n}$ can be identified [Macdonald 1995] with a symmetric polynomial $F_{M}\left(x_{1}, x_{2}, \ldots\right)$ of degree $n$ in infinitely many variables. To each $\mathbb{S}$-module $\mathscr{V}$ we assign the element

$$
F_{\mathscr{V}}\left(x_{1}, \ldots, x_{k}, \ldots\right)=\sum_{n \geq 1} F_{\mathscr{V}(n)}\left(x_{1}, \ldots, x_{k}, \ldots\right)
$$

of the algebra $\Lambda$ of symmetric functions. This algebra is the completion of the algebra of symmetric polynomials in infinitely many variables with respect to the valuation defined by the degree of a polynomial. It is isomorphic to the algebra of formal power series in Newton power sums $p_{1}, \ldots, p_{n}, \ldots$ The series $F_{\mathscr{V}}$ is a generating series for the characters of symmetric groups. Namely, by multiplying the coefficient of $p_{1}^{n_{1}} \ldots p_{k}^{n_{k}}$ by $1^{n_{1}} n_{1} ! \ldots k^{n_{k}} n_{k}$ !, we obtain the value of the character of $\mathscr{V}(n)$ on a permutation whose decomposition into disjoint cycles contains $n_{1}$ cycles of length $1, \ldots, n_{k}$ cycles of length $k$. This definition can be generalised to the case of differential graded modules; for such a module $\mathscr{V}=\bigoplus_{i} \mathscr{V}_{i}$ we set $F_{\mathscr{V}}=\sum_{i}(-1)^{i} F_{\mathscr{V}_{i}}($ the Euler characteristic of $\mathscr{V})$. 
If $\mathscr{V}$ is equipped with an action of a group $G$ commuting with the action of the symmetric groups, then for each $n$ the group $S_{n} \times G$ acts on the space $\mathscr{V}(n)$. In this case, to $\mathscr{V}$ we assign an element of the algebra $\Lambda_{G}$ of symmetric functions over the character ring of $G$ (or, in other words, a character of $G$ ranging over symmetric functions). We denote this element by $F_{\mathscr{V}}\left(x_{1}, \ldots, x_{n}, \ldots ; g\right)$, where $g \in G$.

Remark 2.5. Further in this text we use the following properties of specialisations of our generating functions:

(1) $f_{\mathscr{V}}(x)=\left.F_{\mathscr{V}}\left(p_{1}, \ldots\right)\right|_{p_{1}=x, p_{2}=p_{3}=\cdots=0}$.

(2) $F_{\mathscr{V}}\left(p_{1}, \ldots\right)=\left.F_{\mathscr{V}}\left(p_{1}, \ldots ; g\right)\right|_{g=e}$, where $e$ is the identity element of the group $G$.

(3) for any finite-dimensional vector space $V$ (considered as an $\mathbb{S}$-module concentrated in degree 1),

$$
f_{\mathscr{V}(V)}(x)=\left.F_{\mathscr{V}}\left(p_{1}, \ldots\right)\right|_{p_{1}=x \operatorname{dim} V, p_{2}=x^{2} \operatorname{dim} V, p_{3}=x^{3} \operatorname{dim} V, \ldots},
$$

where $\mathscr{V}(V)=\mathscr{V} \circ V$ and $f_{\mathscr{V}(V)}(x)$ is the generating function for dimensions of the (graded) vector space $\mathscr{V}(V)$.

\section{Functional equation for characters.}

Definition 2.6. Fix $H\left(x_{1}, x_{2}, \ldots ; g\right) \in \Lambda_{G}$. The plethysm corresponding to $H$ (the plethystic substitution of $H$ ) is the algebra homomorphism $F \mapsto F \circ H$ of $\Lambda_{G}$ into itself that is linear over the character ring of $G$ and is defined on symmetric functions by $p_{n} \circ H=H\left(x_{1}^{n}, x_{2}^{n}, \ldots ; g^{n}\right)$.

In particular,

$$
p_{n} \circ\left(H\left(p_{1}, p_{2}, \ldots, p_{k}, \ldots ; g\right)\right)=H\left(p_{n}, p_{2 n}, \ldots, p_{k n}, \ldots ; g^{n}\right) .
$$

Let $\varepsilon$ be the involution of $\Lambda_{G}$ linear over the character ring of $G$ and taking $p_{n}$ to $-p_{n}$.

Theorem 2.7 [Dotsenko and Khoroshkin 2007]. Suppose that the operad $\mathscr{O}$ is Koszul. Then the following equation holds in $\Lambda_{G}$ :

$$
\varepsilon\left(F_{\mathscr{O}}\right) \circ \varepsilon\left(F_{\mathscr{O}} !\right)=p_{1} .
$$

\section{Calculation of dimensions and characters}

Note that the components of $\mathrm{As}^{2}$ and $\left(\mathrm{As}^{2}\right)^{!}$are equipped with an action of $\mathrm{SL}_{2}$ (arising from the action on the space of generators of the operad $\mathrm{As}^{2}$ ), which commutes with the action of the symmetric groups. All information about these operads will follow from the functional equation on the characters and the explicit description of the representation $\left(\mathrm{As}^{2}\right)^{!}(n)$ of the group $\mathrm{SL}_{2} \times S_{n}$. 
The character ring of $\mathrm{SL}_{2}$ is isomorphic to the ring of Laurent polynomials in one variable $q$-for example, the character of the $n$-dimensional irreducible representation $L(n-1)$ is equal to $\left(q^{n}-q^{-n}\right) /\left(q-q^{-1}\right)$. The element of $\Lambda_{\mathrm{SL}_{2}}$ corresponding to an $\mathbb{S}$-module $\mathscr{V}$ is denoted by $F_{\mathscr{V}}\left(p_{1}, \ldots, p_{n}, \ldots ; q\right)$. This notation differs a little from the one introduced above, but we hope that this will not lead to confusion. In this case, the plethysm is defined by $p_{n} \circ q=q^{n}$.

Theorem 3.1. For the operad of two compatible associative products, we have

$$
\begin{gathered}
f_{\mathrm{As}^{2}}(x)=\sum_{n \geq 1} c_{n} x^{n}, \quad F_{\mathrm{As}^{2}}\left(p_{1}, \ldots\right)=\sum_{n \geq 1} c_{n} p_{1}^{n}, \\
F_{\mathrm{As}^{2}}\left(p_{1}, \ldots ; q\right)=\sum_{n \geq 1} p_{1}^{n}\left(q^{n-1}+N_{n, 1} q^{n-3}+\cdots+N_{n, k} q^{n-1-2 k}+\cdots\right),
\end{gathered}
$$

where $c_{n}=\frac{1}{n+1}\left(\begin{array}{c}2 n \\ n\end{array}\right)$ and $N_{n, k}=\frac{1}{n}\left(\begin{array}{l}n \\ k\end{array}\right)\left(\begin{array}{c}n \\ k+1\end{array}\right)$ are the Catalan and Narayana numbers.

Proof. Note that the substitution $p_{1}=x$ transforms the second formula into the first one, and the substitution $q=1$ transforms the third equation into the second (Narayana numbers refine Catalan numbers; see [Stanley 1999]). Thus, the third statement implies the other two, so we shall restrict ourselves to proving only the former. From the results from [Strohmayer 2008] (combined with results from [Dotsenko and Khoroshkin 2007] on $\mathrm{SL}_{2}$-modules), it follows that as an $S_{n} \times \mathrm{SL}_{2}$ module,

$$
\left(\mathrm{As}^{2}\right)^{!}(n)=\mathbb{Q} S_{n} \otimes L(n-1) .
$$

Thus, the $S_{n} \times \mathrm{SL}_{2}$-character of the Koszul dual operad is given by the formula

$$
\sum_{n \geq 1} \frac{q^{n}-q^{-n}}{q-q^{-1}} p_{1}^{n}=\frac{p_{1}}{\left(1-q p_{1}\right)\left(1-q^{-1} p_{1}\right)} .
$$

The functional equation for characters implies that the character

$$
F_{\mathrm{As}^{2}}\left(p_{1}, \ldots, p_{n}, \ldots ; q\right)
$$

satisfies

$$
\frac{F_{\mathrm{As}^{2}}}{\left(1+q F_{\mathrm{As}^{2}}\right)\left(1+q^{-1} F_{\mathrm{As}^{2}}\right)}=p_{1} \text {. }
$$

From this equation it is clear that $F_{\mathrm{As}^{2}}\left(p_{1}, \ldots ; q\right)$ depends only on $p_{1}$ (and $q$ ).

On the other hand, it is well known (see, for example, [Stanley 1999], which should be adjusted to our parametrisation of Narayana numbers) that the generating function

$$
N(t, x)=1+\sum_{n=1}^{\infty} \sum_{k=0}^{n-1} N_{n, k} t^{n} x^{k}
$$


of Narayana numbers satisfies the equation

$$
t x N^{2}(t, x)-t x N(t, x)+t N(t, x)-N(t, x)+1=0 .
$$

It is easy to see that the third statement of the theorem is equivalent to the following equation for generating functions:

$$
N\left(p_{1} q^{-1}, q^{2}\right)=1+q^{-1} F_{\mathrm{As}^{2}}\left(p_{1}, q\right) .
$$

Let

$$
N\left(p_{1} q^{-1}, q^{2}\right)=1+q^{-1} G\left(p_{1}, q\right) .
$$

From the functional (3-2) we deduce that $G$ satisfies

$$
p_{1} q\left(1+q^{-1} G\right)^{2}-p_{1} q\left(1+q^{-1} G\right)+\frac{p_{1}}{q}\left(1+q^{-1} G\right)-\left(1+q^{-1} G\right)+1=0,
$$

which can be rewritten as

$$
p_{1}\left(1+\left(q+q^{-1}\right) G+G^{2}\right)=G .
$$

The latter equation coincides with (3-1), and determines $G$ uniquely; thus $G=F_{\mathrm{As}^{2}}$.

Corollary 3.2. (1) As $S_{n}$-module, $\mathrm{As}^{2}(n)$ is free of rank $c_{n}$.

(2) As $S_{n} \times \mathrm{SL}_{2}$-module,

$$
\mathrm{As}^{2}(n) \simeq \mathbb{Q} S_{n} \otimes\left(L(n-1)+L(n-3)^{N_{n, 1}-1}+L(n-5)^{N(n, 2)-N(n, 1)}+\cdots\right) .
$$

Proof. The $\mathrm{SL}_{2}$-character of the module

$$
L(n-1)+L(n-3)^{N_{n, 1}-1}+L(n-5)^{N(n, 2)-N(n, 1)}+\cdots
$$

is equal to

$$
q^{n-1}+N_{n, 1} q^{n-3}+N_{n, 2} q^{n-5}+\cdots+N_{n, k} q^{n-1-2 k}+\cdots+N_{n, n-1} q^{1-n},
$$

so the second statement follows. The first statement is obtained from the second one, if we forget about the $\mathrm{SL}_{2}$-action.

Corollary 3.3. The dimension of the $k$-th component of $\operatorname{As}^{2}(V)$, the free $\mathrm{As}^{2}$ algebra generated by a vector space $V$, is equal to $c_{k}(\operatorname{dim} V)^{k}$. In particular, the dimension of the $k$-th graded component of the free $\mathrm{As}^{2}$-algebra with one generator is equal to the $k$-th Catalan number.

Proof. This follows immediately from our previous results: we just apply the third formula of Remark 2.5 to $\operatorname{As}^{2}(V)$.

Remark 3.4. The relations in $\mathrm{As}^{2}$ do not change the order of arguments of the operations. This means that this operad is a symmetrisation of a nonsymmetric 
operad, which justifies our observations above that

- $\mathrm{As}^{2}(n)$ is free as an $S_{n}$-module,

- $\operatorname{dim} \operatorname{As}^{2}(V)_{k}=\frac{\operatorname{dim} \operatorname{As}^{2}(k)}{k !}(\operatorname{dim} V)^{k}$, and

- $F_{\mathrm{As}^{2}}\left(p_{1}, \ldots ; q\right)$ depends only on $p_{1}$ (and $q$ ).

(No recourse to the functional equation is necessary, since these observations are reflections of a general fact on symmetrisations of nonsymmetric operads.)

\section{A monomial basis for $\mathrm{As}^{2}$}

In this section we describe a monomial basis for the components of the operad $\mathrm{As}^{2}$. One can compare the methods and structure of this paragraph to the same in [Dotsenko and Khoroshkin 2007] in the case of the operad of two compatible Lie brackets. In this section, we prefer to think of components of our operad in terms of the multilinear elements in free algebras.

Definition 4.1. Given a finite ordered set

$$
A=\left\{a_{1}, a_{2}, \ldots, a_{n}\right\},
$$

with $a_{1}<a_{2}<\cdots<a_{n}$, define a family of monomials $\mathfrak{B}(A)$ in the free algebra $\operatorname{As}^{2}(A)$ recursively. Our recursive definition also assigns to a monomial $m$ its "top level operation" $t(m) \in\{1,2\}$, which is used to define further monomials.

- For $A=\left\{a_{1}\right\}$, let $\mathfrak{B}(A)=\left\{a_{1}\right\}$, and let $t\left(a_{1}\right)=1$.

- For $n>1$, a monomial $b$ belongs to $\mathfrak{B}(A)$ if and only if it satisfies one of the two conditions:

(1) $b=a_{k} \star_{1} b^{\prime}$, where $1 \leq k \leq n$ and $b^{\prime} \in \mathfrak{B}\left(A \backslash\left\{a_{k}\right\}\right)$; in this case we put $t(b)=1$.

(2) $b=b_{1} \star_{2} b_{2}$, where $b_{1} \in \mathfrak{B}\left(A_{1}\right), b_{2} \in \mathfrak{B}\left(A_{2}\right)$ for some $A_{1} \sqcup A_{2}=A$, and $t\left(b_{1}\right)=1$; in this case we put $t(b)=2$.

Theorem 4.2. The family of monomials $\mathfrak{B}(A)$ provides a basis for the multilinear part of the free algebra $\operatorname{As}^{2}(A)$.

Proof. We shall prove that this family spans the multilinear part of $\operatorname{As}^{2}(A)$, and that its number of elements is equal to the dimension of this component - that is, the dimension of $\operatorname{As}^{2}(|A|)$. It will follow that it has to be a basis.

Lemma 4.3. The family of monomials $\mathfrak{B}(A)$ spans the multilinear part of $\operatorname{As}^{2}(A)$.

Proof. Consider a monomial $m$. It is a product of two monomials, and by induction we can assume that they both belong to families $\mathfrak{B}\left(A^{\prime}\right)$ for some sets $A^{\prime} \subset A$. Using 
the associativity property for each of the products, we are left with only one case in which $m$ does not belong to $\mathfrak{B}(A)$, namely

$$
m=\left(m_{1} \star_{2} m_{2}\right) \star_{1} m_{3}
$$

for some $m_{1}, m_{2}, m_{3}$. In this case, we use the compatibility relation (2-1):

$$
m=m_{1} \star_{2}\left(m_{2} \star_{1} m_{3}\right)+m_{1} \star_{1}\left(m_{2} \star_{2} m_{3}\right)-\left(m_{1} \star_{1} m_{2}\right) \star_{2} m_{3},
$$

which shows that we can proceed by induction: in the first two summands, the degree of the first factor has decreased, and the last summand has fewer products of the second type in its first factor.

Lemma 4.4. The number of elements in $\mathfrak{B}(A)$ is equal to $(2|A|) ! /(|A|+1)$ !.

Proof. Let $\beta_{n}=|\mathfrak{B}([n])|$. Moreover, for $i=1,2$ let $\beta_{i, n}=\left|\mathfrak{B}_{i}([n])\right|$, where $\mathfrak{B}_{i}([n])$ is the set of all monomials $b \in \mathfrak{B}([n])$ with $t(b)=i$. We use exponential generating functions again:

$$
\beta(x)=\sum_{l \geq 1} \frac{\beta_{l} x^{l}}{l !}, \quad \beta_{i}(x)=\sum_{l \geq 1} \frac{\beta_{i, l} x^{l}}{l !},
$$

The first condition implies that $\beta_{1, n+1}=(n+1) \beta_{n}$, which can be rewritten as

$$
\beta_{1}(x)-x=x \beta(x) .
$$

The definition of $\mathfrak{B}([n])$ basically means that on the level of $\mathbb{S}$-modules,

$$
\mathfrak{B}_{2}(n)=\left(\operatorname{As} \circ \mathfrak{B}_{1}\right)(n)
$$

for $n \geq 2$, so

$$
\beta_{2}(x)=\left(f_{\text {As }}(x)-x\right) \circ \beta_{1}(x) .
$$

Let us rewrite this equation using the formulae

$$
\beta_{1}(x)+\beta_{2}(x)=\beta(x) \quad \text { and } \quad f_{\mathrm{As}}(x)=\frac{x}{1-x} .
$$

We get

$$
\beta(x)=\frac{\beta_{1}(x)}{1-\beta_{1}(x)}
$$

This can be rewritten as $\beta_{1}(x)=\frac{\beta(x)}{1+\beta(x)}$. Now we can substitute it into (4-1), and get the equation

$$
\frac{\beta(x)}{1+\beta(x)}=x(1+\beta(x)) \text {, }
$$

which coincides with the functional equation for $f_{\mathrm{As}^{2}}(x)$ obtained from (3-1) by setting $q=1$. This concludes the proof of the lemma and of Theorem 4.2. 


\section{Free algebras over $\mathbf{A s}^{2}$}

In this section, we prove that any free algebra with two compatible products is free as an associative algebra. Let us recall a theorem which is one of the main ingredients in our proof.

A criterion for free algebras. Let $\mathscr{P}$ be an operad. Assume that $\mathscr{P}(1)=\mathbb{Q}$ and let $\mathscr{P}^{+}$be the $\mathbb{S}$-module such that $\mathscr{P}=\mathscr{P}(1) \oplus \mathscr{P}^{+}$. Let $\mathscr{A}$ be a $\mathscr{P}$-algebra in the category of $\mathbb{S}$-modules. The structure of a $\mathscr{P}$-algebra on $\mathscr{A}$ is given by a morphism $\mu: \mathscr{P} \circ \mathscr{A} \rightarrow \mathscr{A}$.

Let us define a decreasing $\mathscr{P}$-algebra filtration of $\mathscr{A}$ : for each $k \geq 0$ we a define

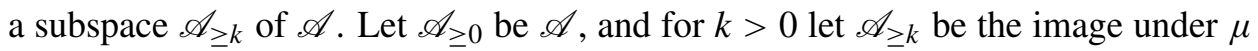
of $\mathscr{P}^{+} \circ \mathscr{A}_{\geq k-1}$.

We will assume that this filtration is separating, which is true, for instance, if $\mathscr{A}$ has a grading concentrated in positive degrees.

Let us define $H_{0}(\mathscr{A})$ to be the degree 0 component $\mathscr{A}_{\geq 0} / \mathscr{A}_{\geq 1}$ of the associated graded $\mathscr{P}$-algebra gr $\mathscr{A}$.

Let us choose a section of $H_{0}(\mathscr{A})$ in $\mathscr{A}$. Consider $\mathscr{P}\left(H_{0}(\mathscr{A})\right)$, that is the free $\mathscr{P}$-algebra generated by $H_{0}(\mathscr{A})$. Then there exists a unique morphism $\theta$ of $\mathscr{P}$-algebras from $\mathscr{P}\left(H_{0}(\mathscr{A})\right)$ to $\mathscr{A}$ extending the chosen section.

Theorem 5.1 [Chapoton 2007; Fresse 1998]. The morphism $\theta$ is surjective. Therefore, if dimensions (or graded characters) of $\mathscr{A}$ and $\mathscr{P}\left(H_{0}(\mathscr{A})\right)$ are equal, then $\theta$ is an isomorphism.

\section{Free algebras with two compatible products are free.}

Theorem 5.2. Free algebras with two compatible brackets are free as associative algebras.

Proof. Let us first prove that there exists an $\mathbb{S}$-module $\mathscr{G}$ such that the $\mathbb{S}$-modules $\mathrm{As}^{2}$ and As o $\mathscr{G}$ are isomorphic. To do that, we apply the above criterion for free algebras in the case $\mathscr{P}=$ As and $\mathscr{A}=\mathrm{As}^{2}$, where the $\mathscr{P}$-algebra structure is given by the second product. This means that we should put $\mathscr{G}:=H_{0}(\mathscr{A})$, and in order to prove our theorem, we only need to prove that graded characters of $\mathrm{As}^{2}$ and As o $\mathscr{G}$ are equal. This is guaranteed by the next lemma.

Lemma 5.3. (1) For each component of $\mathrm{As}^{2}$, the part of its monomial basis consisting of elements $b$ with $t(b)=1$ can be taken as a lift $\theta: \mathscr{G} \rightarrow \mathrm{As}^{2}$;

(2) $f_{\mathrm{As}^{2}}(x)=f_{\mathrm{As}} \circ f_{\mathscr{G}}(x)$.

Proof. From our proof of the spanning property, it follows that any monomial for which the top level operation is the second product belongs to the subspace spanned by all basis elements $b$ with $t(b)=2$, so the quotient by the space of all such monomials is identified with the complementary subspace. 
Also, the (4-2) is

$$
f_{\mathrm{As}^{2}}(x)=f_{\mathrm{As}} \circ \beta_{1}(x),
$$

which is exactly what our second statement claims.

Now we are ready to prove our theorem. For a vector space $V$,

$$
\operatorname{As}^{2}(V) \simeq \operatorname{As}(\mathscr{G}(V)),
$$

so the free $\mathrm{As}^{2}$-algebra with generators $V$ is isomorphic to the free associative algebra with generators $\mathscr{G}(V)$.

\section{Labelled rooted trees and compatible products}

A planar rooted tree is an abstract rooted tree with a linear order on the set of children of every vertex. Alternatively, one can imagine a tree embedded into the plane in such a way that all children of any vertex $v$ have their $y$-coordinates less than the $y$-coordinate of $v$ (in this case, the linear order appears from reading the outgoing edges from left to right).

Proposition 6.1 [Stanley 1999]. The number of planar rooted trees with $n+1$ vertices is equal to the Catalan number $c_{n}$.

Thus, if we consider the planar rooted trees with $k+1$ vertices equipped with a labelling of all nonroot vertices by elements of some finite set $S$, the number of these objects is equal to $c_{k}(\# S)^{k}$, which is, by Corollary 3.3, equal to the dimension of the $k$-th component of the free $\mathrm{As}^{2}$-algebra generated by $S$. In the remaining part of this section, we show that this fact is not a mere coincidence. Namely, we define two compatible associative products on the linear span of all planar rooted trees with $S$-labeled nonroot vertices, and show that this linear span is free as an $\mathrm{As}^{2}$-algebra.

Denote by $\operatorname{RT}(S)$ the collection of all planar rooted trees whose nonroot vertices are labeled by elements of a finite set $S$ (possibly with repeated labels). We start by defining several operations on the linear span $\mathbb{Q} \mathrm{RT}(S)$.

Definition 6.2. Let $T_{1}, T_{2} \in \mathrm{RT}(S)$. Define the tree $T_{1} \cdot T_{2}$ as the tree obtained by identifying the roots of $T_{1}$ and $T_{2}$; the linear ordering of the children of this vertex is uniquely defined by the condition that all children coming from $T_{1}$ precede all children coming from $T_{2}$. This operation is associative, and every $T \in \operatorname{RT}(S)$ whose root has $k$ children can be uniquely decomposed as $T=T[1] \cdot T[2] \cdots \cdot T[k]$, where for each $j$ the root of the tree $T[j]$ has only one child.

Let us denote by Vertices $(T)$ the set of all vertices of a tree $T \in \mathrm{RT}(S)$ (including the root), and by $\operatorname{Internal}(T)$ the set of all internal vertices of $T$.

Definition 6.3. Let $T_{1}, T_{2} \in \mathrm{RT}(S)$. Assume that the root of $T_{1}$ has $k$ children (as in Definition 6.2). 
(1) To every mapping $f:[k] \rightarrow \operatorname{Vertices}\left(T_{2}\right)$ we assign a new tree $T_{1} \circ^{f} T_{2}$ which is obtained as follows. For each $v \in \operatorname{Vertices}\left(T_{2}\right)$ we let

$$
f^{-1}(v)=\left\{i_{1}<\cdots<i_{s}\right\},
$$

form a tree $T_{1}\left[i_{1}\right] \cdots T_{1}\left[i_{s}\right]$, and identify the root of this tree with the vertex $v$ (keeping the label of $v$ ) in a way that all children of this tree are placed left of all the children of $v$ in $T_{2}$.

(2) To every mapping $g:[k] \rightarrow \operatorname{Internal}\left(T_{2}\right)$ we assign a new tree $T_{1} \circ_{g} T_{2}$ which is obtained as follows. For each $v \in \operatorname{Internal}\left(T_{2}\right)$ we let

$$
g^{-1}(v)=\left\{j_{1}<\cdots<j_{r}\right\},
$$

form a tree $T_{1}\left[j_{1}\right] \cdots T_{1}\left[j_{r}\right]$, and identify the root of this tree with the vertex $v$ (keeping the label of $v$ ) in a way that all children of this tree are placed left of all the children of $v$ in $T_{2}$.

We now define two products on $\mathbb{Q R T}(S)$.

Definition 6.4. Let $T_{1}, T_{2} \in \mathrm{RT}(S)$. Assume that the root of $T_{1}$ has $k$ children. Define the products $T_{1} \star_{1} T_{2}$ and $T_{1} \star_{2} T_{2}$ by

$$
\begin{aligned}
T_{1} \star_{1} T_{2} & =\sum_{f:[k] \rightarrow \operatorname{Vertices}\left(T_{2}\right)} T_{1} \circ^{f} T_{2}, \\
T_{1} \star_{2} T_{2} & =\sum_{g:[k] \rightarrow \operatorname{Internal}\left(T_{2}\right)} T_{1} \circ_{g} T_{2} .
\end{aligned}
$$

Example 6.5. For the trees

$$
T_{1}=\left.\right|_{a} \quad T_{2}=\bigwedge_{b}
$$

the product $T_{1} \star_{1} T_{2}$ is equal to
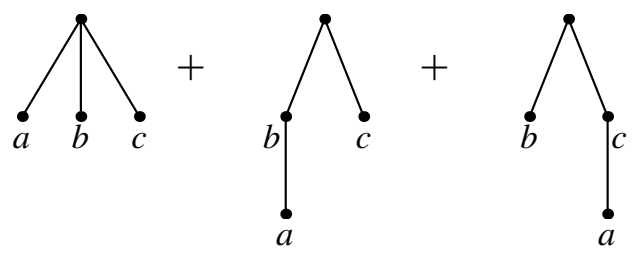

while the product $T_{1} \star_{2} T_{2}$ is equal to

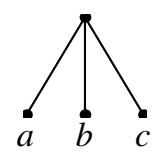


Theorem 6.6. (1) The products $\star_{1}$ and $\star_{2}$ are associative and compatible with each other.

(2) The $\mathrm{As}^{2}$-algebra $\mathbb{Q} \mathrm{RT}(S)$ is isomorphic to the free $\mathrm{As}^{2}$-algebra generated by $S$.

Example 6.7. For the trees

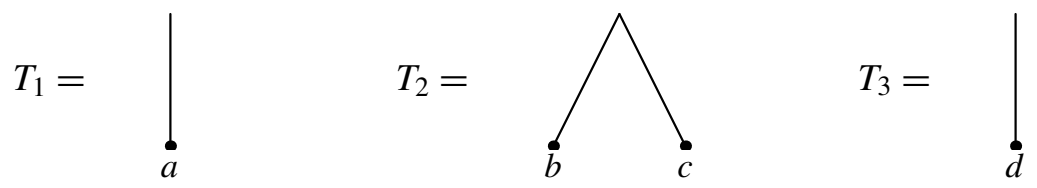

the four products that occur in the compatibility relation (2-1) are as follows. $T_{1} \star_{1}\left(T_{2} \star_{2} T_{3}\right):$

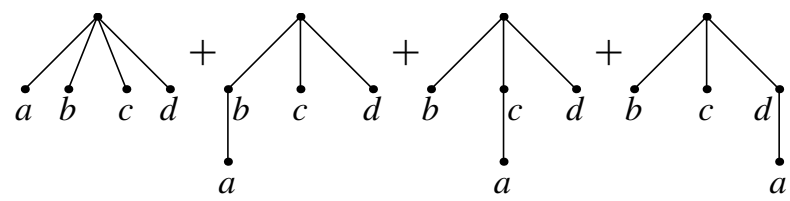

$T_{1} \star_{2}\left(T_{2} \star_{1} T_{3}\right):$

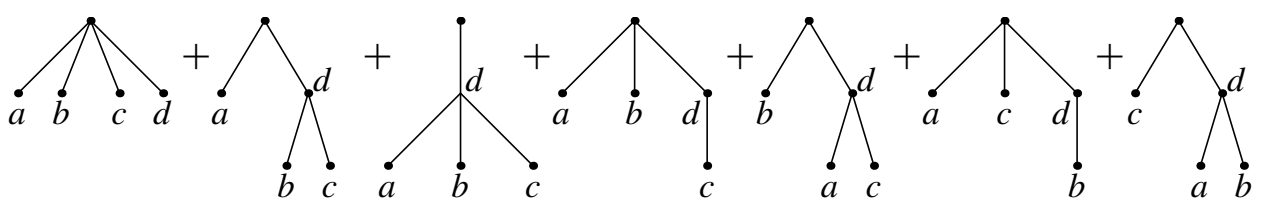

$\left(T_{1} \star_{2} T_{2}\right) \star_{1} T_{3}:$

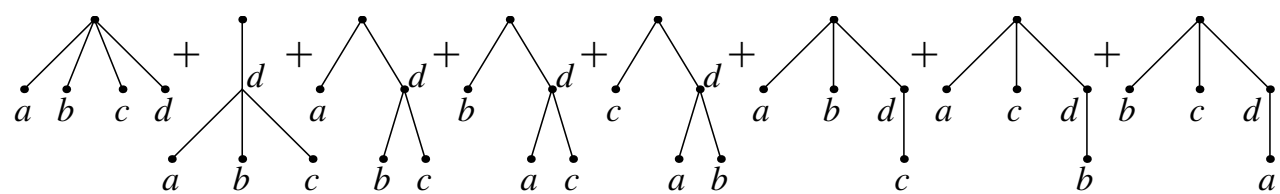

$\left(T_{1} \star_{1} T_{2}\right) \star_{2} T_{3}:$

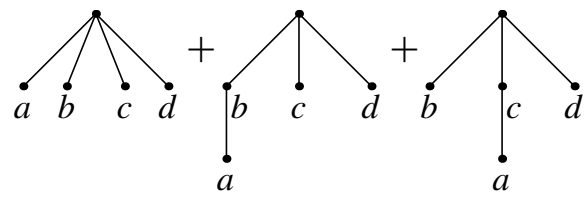

Thus the compatibility condition is satisfied.

Proof. The associativity conditions for both products are pretty transparent; to show that all the terms in consecutive product $\Pi_{1}=T_{1} \star_{1}\left(T_{2} \star_{1} T_{3}\right)$ appear in the product $\Pi_{2}=\left(T_{1} \star_{1} T_{2}\right) \star_{1} T_{3}$ one should just notice that to obtain the terms in $\Pi_{1}$ where subtrees of $T_{1}$ are attached directly to vertices of $T_{3}$ (all other terms appear in $\Pi_{2}$ 
for tautological reasons) we should just attach the corresponding subtrees to the root of $T_{2}$ when computing $T_{1} \star_{1} T_{2}$ for $\Pi_{2}$, then we can attach them as required when computing the final product. The same argument works for the second product.

We shall establish the compatibility condition rewritten in the form

$$
\left(T_{1} \star_{2} T_{2}\right) \star_{1} T_{3}-T_{1} \star_{2}\left(T_{2} \star_{1} T_{3}\right)=T_{1} \star_{1}\left(T_{2} \star_{2} T_{3}\right)-\left(T_{1} \star_{1} T_{2}\right) \star_{2} T_{3} .
$$

The reason is that for our products both the left hand side and the right hand side are combinations of trees with nonnegative coefficients, and we can interpret the summands that appear there in a rather nice and simple way. Namely, the trees that appear on the left hand side are those for which there exist subtrees of $T_{1}$ that are attached to some leaves of $T_{3}$. Obviously, the left hand side has the same interpretation. Details are simple and we leave them to the reader.

Now we shall prove that the algebra $\mathbb{Q} \mathrm{RT}(S)$ is free as an $\mathrm{As}^{2}$-algebra. Note that this algebra admits a natural grading by the number of nonroot vertices of a tree, and the dimensions of graded components are precisely the dimensions of the graded components of the free $\mathrm{As}^{2}$-algebra generated by $S$. It remains to show that our algebra is generated as an $\mathrm{As}^{2}$-algebra by elements of degree 1; it will follow that it is a quotient of the corresponding free algebra, and since it has the same dimensions of graded components, these two algebras should be isomorphic. Thus, it remains to prove the following lemma.

Lemma 6.8. As an $\mathrm{As}^{2}$-algebra, $\mathbb{Q} \mathrm{RT}(S)$ is generated by elements of degree 1 .

Proof. We use induction on degree. Assume that all trees of degree at most $k$ are products of elements of degree 1 . We show that the same holds for trees of degree $k+1$. For a tree $T$, let us call the children of the rightmost child of the root the principal grandchildren of the root; denote the number of principal grandchildren by $\operatorname{pg}(T)$. Let us prove the step of induction using the induction on $\operatorname{pg}(T)$.

For $\operatorname{pg}(T)=0$, the rightmost child of the root is a leaf. Denote by $s$ the label of that leaf, and by $T^{\prime}$ the tree obtained from $T$ by deleting the rightmost child of the root. Then

$$
T^{\prime} \star_{2} T(s)=T,
$$

where $T(s)$ denotes the tree with two vertices whose nonroot vertex is labeled by $s$. The degree of $T^{\prime}$ is less, so our statement follows.

For $\operatorname{pg}(T)=k$, let us denote by $T_{1}$ the tree obtained from $T$ by deleting all principal grandchildren of the root (and the trees they are the roots of), but keeping the rightmost child of the root (and its label). Also, denote by $T_{2}$ the complementary tree, that is, the subtree whose root is the rightmost child of the root of $T$ (with its label deleted). Then the tree expansion of $T_{2} \star_{1} T_{1}$ consists of $T$ and a combination of other trees $\widetilde{T}$ for which $\operatorname{pg}(\widetilde{T})<k$, so we can proceed by induction. 
Remark 6.9. Theorem 6.6 can be used to obtain an alternative proof of one of the main results in [Grossman and Larson 1989]. Namely, since the first product $T_{1} \star_{1} T_{2}$ is the Grossman-Larson product on $\mathbb{Q R T}(S)$, it follows from Theorem 5.2 that the Grossman-Larson algebra of planar rooted trees is a free associative algebra; moreover, from our proofs it is easy to see that as a generating set of this algebra we can take all trees whose root has only one child. These are exactly the results of Grossman and Larson.

\section{Remarks and open questions}

Relation to the Grossman-Larson Hopf algebra structure. Recall that Grossman and Larson [1989] introduced an algebra of planar rooted trees as a Hopf algebra with the coproduct defined as follows.

Definition 7.1 [Grossman and Larson 1989]. Define the coproduct

$$
\Delta: \mathbb{Q R T}(S) \rightarrow \mathbb{Q R T}(S) \otimes \mathbb{Q R T}(S)
$$

by the formula

$$
\Delta(T)=\sum_{I \sqcup J=[k]} T\left[i_{1}\right] \cdots \cdot T\left[i_{p}\right] \otimes T\left[j_{1}\right] \cdots \cdot T\left[j_{q}\right],
$$

where $T=T[1] \cdots T[k] \in \mathbb{Q} \mathrm{RT}(S)$, and the notation $I=\left\{i_{1}<\cdots<i_{p}\right\}$, $J=\left\{j_{1}<\cdots<j_{q}\right\}$ is used.

One could ask what is the relation between this coproduct and the second product that we introduced.

Proposition 7.2. Consider $\mathbb{Q} \mathrm{RT}(S)$ as an associative algebra with respect to either of the products $\star_{1}, \star_{2}$ (and introduce the product on its tensor square accordingly). Then $\Delta$ is an algebra homomorphism.

Proof. For the first product, this statement is proved in [Grossman and Larson 1989]. For the second product, one can use the same proof with some slight modifications (basically, what should be done is simply forgetting all summands where grafting to leaves occurs).

Remark 7.3. It is worth mentioning that although the tensor product of two $\mathrm{As}^{2}$ algebras can be turned into an $\mathrm{As}^{2}$-algebra in many different ways, two products on the tensor square of the free algebra that we just described are not compatible; the family of products

$$
\left(a_{1} \otimes b_{1}\right) \star_{\lambda, \mu}\left(a_{2} \otimes b_{2}\right)=\left(\lambda a_{1} \star_{1} a_{2}+\mu a_{1} \star_{2} a_{2}\right) \otimes\left(\lambda b_{1} \star_{1} b_{2}+\mu b_{1} \star_{2} b_{2}\right)
$$

is a pencil of associative products, but it is not a linear pencil anymore (they rather resemble pencils of associative products from [Moerdijk 2001]). Thus, the 
relationship between Hopf algebra structure and the structure of an algebra with two compatible products is yet to be clarified.

Relation to other operads realised by planar trees. The following observation is due to Loday (private communication).

Remark 7.4. Consider the operad $\mathscr{O}_{q}$ generated by two binary operations $\circ$ and $\bullet$ which satisfy the relations

$$
\begin{aligned}
(x \circ y) \circ z & =x \circ(y \circ z), \\
(x \bullet y) \circ z+q(x \circ y) \bullet z & =x \bullet(y \circ z)+q x \circ(y \bullet z), \\
(x \bullet y) \bullet z & =x \bullet(y \bullet z) .
\end{aligned}
$$

Then $\mathscr{O}_{0}$ is the operad Dup of duplicial algebras [Loday 2008], while $\mathscr{O}_{1}$ is the operad $\mathrm{As}_{2}$.

Also, consider the operad $\mathscr{P}_{t}$ generated by two binary operations $\prec$ and $\succ$ which satisfy the relations

$$
\begin{gathered}
(x \prec y) \prec z=x \prec(y \prec z)+t x \prec(y \succ z), \\
(x \succ y) \prec z=x \succ(y \prec z), \\
(x \succ y) \succ z+t(x \prec y) \succ z=x \succ(y \succ z) .
\end{gathered}
$$

Then $\mathscr{P}_{0}$ is the operad Dup, while $\mathscr{P}_{1}$ is the operad Dend of dendriform algebras [Loday 2008].

It is known that free algebras over Dend and Dup can be realised by planar trees. It would be interesting to define in a pure combinatorial way a 2-parameter family of pairs of binary operations $\star_{1, q, t}$ and $\star_{2, q, t}$ on $\mathbb{Q R T}(S)$ which have correct specialisations to $q=t=0$ (duplicial case) $q=1, t=0$ (compatible associative products) and $q=0, t=1$ (dendriform case).

Other Hopf-algebraic families of trees. Some general phenomenon that we think is worth mentioning here is the existence of compatible associative products for many other well known algebras where the product is described via combinatorics of trees. The main idea is very simple. If the product in the linear span of rooted trees (planar or not) is defined for two trees $T_{1}=T_{1}[1] \cdot T_{1}[2] \cdots T_{1}[k]$ and $T_{2}$ as the sum of all graftings of some type of trees $T_{1}[i]$ to vertices of the tree $T_{2}$, then another product over all graftings of the same type but only to internal vertices is compatible with the first product. For algebras of planar binary trees (which also often occur in literature) an analogous recipe holds: if a product is defined in terms of graftings, then graftings only to the "left-going" leaves produce a compatible product. 
Example 7.5. The Connes-Kreimer Hopf algebra of renormalisation is a polynomial algebra on (abstract) rooted trees, or, in other words, an algebra on the linear span of forests of rooted trees [Connes and Kreimer 1998]. If we take its dual, and identify the dual of each forest with the rooted tree having all trees of the forest grafted at its root vertex, the coproduct of Connes and Kreimer yields a product on the linear span of rooted trees which is defined in terms of graftings as above. Thus, this algebra is naturally endowed with another product which is compatible with the original one.

Example 7.6. Similarly, consider the noncommutative Connes-Kreimer Hopf algebra NCK of Foissy [2002a; 2002b], which is a free associative algebra on planar rooted trees, or, in other words, an algebra on the linear span of (ordered) forests of planar rooted trees. If we take its dual, and identify the dual of each forest with the planar rooted tree having all trees of the forest grafted at its root vertex, the coproduct of Foissy leads to another product on $\mathbb{Q R T}(S)$ which is again defined in terms of graftings. It follows that $N C K$ has a natural structure of an algebra with two compatible products. Results of Foissy on isomorphisms of Hopf algebras also produce compatible products on some other algebras on trees, for example, the Brouder-Frabetti [2003] Hopf algebra of renormalisation in QED.

One can easily check that unlike the case of the Grossman-Larson product, the dual of the Foissy algebra is not a free algebra with two compatible products; for example, the $\star_{2}$-subalgebra of $N C K$ generated by elements of degree 1 (that is, trees with one leaf) is commutative. We expect that this is in some sense the only obstruction to freeness.

Conjecture 7.7. As ${ }^{2}$-subalgebra of NCK generated by elements of degree 1 is a free algebra over the operad of two compatible associative products one of which is, in addition, commutative.

The operad that shows up here does not seem to have many good properties. In particular, it is not Koszul, and not much is known about the growth of dimensions of its components.

It was pointed out by the referee that our results in Section 6 carry a certain resemblance with those of Patras and Schocker [2008], who studied the Hopf algebra structure on the linear span of set compositions (the twisted descent algebra). They prove that certain combinatorially defined algebras are free; labeled planar trees (with some restrictions on labels) do appear in their work, they also define two products on their trees for which the difference of two products is a combination of trees with nonnegative integer coefficients (just as in our case). However, their products are not compatible, and we do not know whether there is any relation of our results to those of Patras and Schocker. 
General compatible structures. It is natural to ask which of our results have analogues for other operads of compatible structures (see [Strohmayer 2008] for the formal definition of compatible $\mathscr{O}$-structures for any operad $\mathscr{O}$ ). For the operad of compatible Lie brackets, we can prove an exact analogue of Theorem 5.2: free algebras over that operad are free as Lie algebras. The proof is similar to the proof for compatible associative structures and also makes use of an appropriate monomial basis. We expect that actually both of these statements are particular cases of a very general theorem.

The following conjecture consists of two parts. The first part generalises the main theorem of Strohmayer [2008], while the second one suggests that our theorem also holds in that generality.

Conjecture 7.8. (1) Let $\mathscr{O}$ be a Koszul operad. Then the operad $\mathscr{O}^{2}$ of two compatible $\mathscr{O}$-structures is also Koszul.

(2) If the operad $\mathscr{O}$ is Koszul, then free $\mathscr{O}^{2}$-algebras are free as $\mathscr{O}$-algebras.

\section{Acknowledgements}

This paper was inspired by a table-talk at the Workshop on Algebraic Structures in Geometry and Physics (Leicester, July 2008). The author is grateful to Alexander Odesskii for his questions on the operad of compatible associative products, and to Andrey Lazarev for an opportunity to attend the workshop. He also wishes to thank Alexander Frolkin who read the preliminary version of this article and corrected some misprints and flaws in typesetting and language. Special thanks are to Jean-Louis Loday for useful comments. The author is also indebted to the anonymous referee for informing him of a flaw in the original proof of Lemma 6.8.

\section{References}

[Brouder and Frabetti 2003] C. Brouder and A. Frabetti, "QED Hopf algebras on planar binary trees", J. Algebra 267:1 (2003), 298-322. MR 2004m:81160 Zbl 1056.16026

[Chapoton 2007] F. Chapoton, "Free pre-Lie algebras are free as Lie algebras", preprint, 2007. To appear in Can. Math. Bull. arXiv math/0704.2153

[Connes and Kreimer 1998] A. Connes and D. Kreimer, "Hopf algebras, renormalization and noncommutative geometry”, Comm. Math. Phys. 199 (1998), 203-242. MR 99h:81137 Zbl 0932.16038

[Dotsenko and Khoroshkin 2007] V. V. Dotsenko and A. S. Khoroshkin, "Character formulas for the operad of a pair of compatible brackets and for the bi-Hamiltonian operad", Funktsional. Anal. $i$ Prilozhen. 41:1 (2007), 1-22, 96. In Russian; translated in Funct. Anal. and Appl., 41:1 (2007), 1-17. MR 2008d:18006 Zbl 1145.18001

[Foissy 2002a] L. Foissy, "Les algèbres de Hopf des arbres enracinés décorés. I", Bull. Sci. Math. 126:3 (2002), 193-239. MR 2003d:16049 Zbl 1013.16026

[Foissy 2002b] L. Foissy, "Les algèbres de Hopf des arbres enracinés décorés. II", Bull. Sci. Math. 126:4 (2002), 249-288. MR 2003e:16047 Zbl 1013.16027 
[Fresse 1998] B. Fresse, "Cogroups in algebras over an operad are free algebras", Comment. Math. Helv. 73:4 (1998), 637-676. MR 99i:18010 Zbl 0929.16033

[Ginzburg and Kapranov 1994] V. Ginzburg and M. Kapranov, "Koszul duality for operads", Duke Math. J. 76:1 (1994), 203-272. MR 96a:18004 Zbl 0855.18006

[Grossman and Larson 1989] R. Grossman and R. G. Larson, "Hopf-algebraic structure of families of trees", J. Algebra 126:1 (1989), 184-210. MR 90j:16022 Zbl 0717.16029

[Loday 2008] J.-L. Loday, Generalized bialgebras and triples of operads, Astérisque 320, Soc. math. de France, Paris, 2008. MR 2504663

[Macdonald 1995] I. G. Macdonald, Symmetric functions and Hall polynomials, 2nd ed., The Clarendon Press Oxford University Press, New York, 1995. MR 96h:05207 Zbl 0824.05059

[Markl et al. 2002] M. Markl, S. Shnider, and J. Stasheff, Operads in algebra, topology and physics, Mathematical Surveys and Monographs 96, American Mathematical Society, Providence, RI, 2002. MR 2003f:18011 Zbl 1017.18001

[Moerdijk 2001] I. Moerdijk, "On the Connes-Kreimer construction of Hopf algebras”, pp. 311-321 in Homotopy methods in algebraic topology (Boulder, CO, 1999), edited by J. P. C. Greenlees et al., Contemp. Math. 271, Amer. Math. Soc., Providence, RI, 2001. MR 2002c:18006 Zbl 0987.16032

[Odesskii and Sokolov 2006] A. Odesskii and V. Sokolov, "Algebraic structures connected with pairs of compatible associative algebras", Int. Math. Res. Not. 2006:19 (2006), Art. ID 43734. MR 2007g:16045 Zbl 1151.16030

[Patras and Schocker 2008] F. Patras and M. Schocker, "Trees, set compositions and the twisted descent algebra", J. Algebraic Combin. 28:1 (2008), 3-23. MR 2420777 Zbl 05566021

[Stanley 1999] R. P. Stanley, Enumerative combinatorics, vol. 2, Cambridge Studies in Advanced Mathematics 62, Cambridge University Press, 1999. MR 2000k:05026 Zbl 0928.05001

[Strohmayer 2008] H. Strohmayer, "Operads of compatible structures and weighted partitions", J. Pure Appl. Algebra 212:11 (2008), 2522-2534. MR 2440264 Zbl 1149.18006

Communicated by Mikhail M. Kapranov

Received 2008-09-16 Revised 2009-02-07 Accepted 2009-06-16

vdots@maths.tcd.ie

Dublin Institute for Advanced Studies, 10 Burlington Road, Dublin 4, Ireland http://www. maths.tcd.ie/ vdots 